\title{
El género femenino es un predictor independiente de la mortalidad intrahospitalaria en pacientes con infarto agudo de miocardio con elevación del segmento ST revascularizado mediante una angioplastia primaria
}

\author{
J.J. FERRER-HITAa, A. DOMÍNGUEZ-RODRÍGUEZa , M.J. GARCÍA-GONZÁLEZa, \\ P. ABREU-GONZÁLEZ ${ }^{b}$, S. SAMIMI-FARDa Y F. MARRERO-RODRÍGUEZa
}

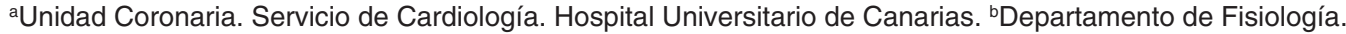
Facultad de Medicina. Universidad de La Laguna. Santa Cruz de Tenerife. España.

Objetivo. Analizar la influencia del sexo en el pronóstico intrahospitalario del infarto agudo de miocardio con elevación del segmento ST (IAMEST) revascularizado mediante una angioplastia primaria (AP).

Diseño y ámbito. Estudio prospectivo de una cohorte de pacientes consecutivamente ingresados en la Unidad Coronaria de un hospital terciario durante el periodo de enero a octubre de 2004, con diagnóstico de IAMEST y tratados con AP.

Pacientes. Muestra consecutiva de 86 pacientes con dicho diagnóstico dividida en dos grupos en función del sexo: $\mathbf{5 2}$ hombres y $\mathbf{3 4}$ mujeres.

Variables de interés principales. En ambos grupos analizamos las características clínico-demográficas basales, la extensión de la enfermedad coronaria (EEC), el éxito de la AP, la aparición de insuficiencia cardiaca (IC) y la mortalidad intrahospitalaria en los primeros 28 días tras el ingreso. Se analizaron predictores de mortalidad en un modelo multivariado.

Resultados. Las mujeres presentaron mayor edad (70 \pm 8 frente a $65 \pm 11 ; p=0,02)$ y prevalencia de diabetes $(37 \%$ frente a $18 \% ; p=0,002)$ y de

Correspondencia: Dr. J.J. Ferrer-Hita.

Servicio de Cardiología. Hospital Universitario de Canarias. Ctra. La Cuesta-Taco, s/n.

38320 Ofra. La Laguna. Santa Cruz de Tenerife. España.

Correo electrónico: juliojferrer@hotmail.com

Manuscrito aceptado el 16-III-2007. hipertensión arterial (58\% frente a 37\%; $p<0,001)$ que los hombres, mientras que estos presentaron mayor frecuencia de tabaquismo (34\% frente a $22 \% ; p=0,001)$. No hubo diferencias en la presencia de hiperlipidemia, la EEC o el éxito de la AP. Las mujeres presentaron mayor incidencia de IC al ingreso $(22 \%$ frente a $12 \% ; p=0,01)$ y de mortalidad intrahospitalaria (17\% frente a $8 \% ; p=$ 0,002). En el análisis multivariado, el sexo femenino y la IC al ingreso permanecieron como predictores de mortalidad intrahospitalaria.

Conclusiones. En nuestro estudio, el sexo femenino fue un predictor independiente de mortalidad intrahospitalaria en pacientes con IAMEST tratados con AP.

PALABRAS CLAVE: infarto de miocardio, angioplastia coronaria transluminal percutánea, factores sexuales, pronóstico.

FEMALE GENDER IS AN INDEPENDENT

PREDICTOR OF IN-HOSPITAL MORTALITY IN PATIENTS WITH ST SEGMENT ELEVATION ACUTE MYOCARDIAL INFARCTION TREATED WITH PRIMARY ANGIOPLASTY

Objective. The aim of this study was to determine the influence of gender on in hospital outcome in patients with ST-segment elevation myocardial infarction (STEMI) who underwent primary angioplasty (PA).

Design and scope. Prospective study of a cohort of patients consecutively admitted to the Coronary Unit of a tertiary hospital in the period of January to October 2004 with the diagnoses of IAMEST and treated with PA. 
FERRER-HITA JJ ET AL. EL GÉNERO FEMENINO ES UN PREDICTOR INDEPENDIENTE DE LA MORTALIDAD INTRAHOSPITALARIA EN PACIENTES CON INFARTO AGUDO DE MIOCARDIO CON ELEVACIÓN DEL SEGMENTO ST REVASCULARIZADO MEDIANTE UNA ANGIOPLASTIA PRIMARIA

Patients. Consecutive sample of 86 patients with this diagnosis divided into two groups based on sex: 52 men and 34 women.

Main variables of interest. In both groups, we analyzed the baseline clinical-demographic characteristics, extension of the coronary disease (ECD), success of the PA, appearance of heart failure (HF) and in-hospital mortality in the first 28 days after admission. We analyzed predictors of mortality in a multivariate model.

Results. The women were older $(70 \pm 8$ versus $65 \pm 11 ; p=0.02$ ) and had greater prevalence of diabetes $(37 \%$ versus $18 \% ; p=0.002)$ and hypertension $(58 \%$ versus $37 \%$; $p<0.001)$ than the men while the men had greater frequency of smoking ( $34 \%$ versus $22 \%$; $p=0.001$ ). There were no differences in the presence of hyperlipidemia, ECD or the success of PA. Women had a higher incidence of HF on admission $(22 \%$ versus $12 \% ; p=$ $0.01)$ and in-hospital mortality $(17 \%$ versus $8 \%$; $p$ $=0.002$ ). In the multivariate analyses, female sex and HF on admission continued to be predictors of in-hospital mortality.

Conclusions. In our study, female gender was an independent predictor of in-hospital mortality in patients with IAMEST treated with PA.

KEY WORDS: myocardial infarction, percutaneous transluminal coronary angioplasty, sexual factors, prognosis.

\section{INTRODUCCIÓN}

Las enfermedades cardiovasculares son la primera causa de mortalidad tanto en hombres como en mujeres en los países desarrollados ${ }^{1,2}$. De entre sus diversas formas de presentación, la cardiopatía isquémica es la principal responsable de la mayoría de estas muertes $^{1,2}$. Afortunadamente, la mortalidad del infarto agudo de miocardio con elevación del segmento ST (IAMEST) ha disminuido considerablemente durante las últimas dos décadas, gracias fundamentalmente a la aplicación sistemática de las diferentes estrategias precoces de reperfusión ${ }^{3-6}$. Sin embargo, mientras la muerte por cardiopatía isquémica ha disminuido en los varones, su incidencia permanece prácticamente estable en las mujeres ${ }^{1,2,6}$.

En la década pasada, diversos estudios analizaron la influencia de las diferencias de sexo en el pronóstico tras un infarto agudo de miocardio. Pese a la profusión de trabajos que se centraron en este aspecto, finalmente no quedó claro si el pronóstico a corto plazo del infarto agudo de miocardio era peor en la mujeres que en los hombres de forma independiente ${ }^{7,8}$. Así, algunos grupos comunicaron una mayor mortalidad en mujeres ${ }^{7,9-11}$ y otros no ${ }^{12-14}$.

A pesar de todo el interés suscitado por el tema en la década pasada y de la epidemiología actual de las enfermedades cardiovasculares y de la cardiopatía isquémica en la mujer, actualmente existe una clara disociación entre la magnitud del problema y la per- cepción real del mismo por parte de todos los profesionales implicados. Ello ha motivado que las diferentes sociedades científicas hayan vuelto a insistir en la importancia de una mayor profundización e implicación en dicho asunto con el fin de mejorar las perspectivas actuales ${ }^{6,15}$.

En este sentido, el objetivo de este estudio fue analizar el valor pronóstico del sexo femenino sobre la mortalidad intrahospitalaria, en los primeros 28 días tras el ingreso, de pacientes con diagnóstico IAMEST y revascularizados con angioplastia primaria $(\mathrm{AP})$ en un hospital de tercer nivel.

\section{PACIENTES Y MÉTODOS}

Se estudió de forma prospectiva una cohorte de 86 pacientes que ingresaron de forma consecutiva en la Unidad Coronaria del Servicio de Cardiología de un hospital terciario, con diagnóstico de IAMEST durante el periodo entre enero y octubre de 2004, y que fueron revascularizados con AP. El diagnóstico de IAMEST se estableció según los criterios publicados en la literatura ${ }^{16}$.

Todos los pacientes fueron sometidos a revascularización percutánea con AP de acuerdo con el programa de 24 horas disponible en nuestro hospital. En la población de estudio se establecieron dos grupos de comparación en función del sexo.

En ambos grupos se analizaron las siguientes características clínico-demográficas basales: edad y factores de riesgo cardiovascular como historia de tabaquismo, diabetes mellitus (DM), hipertensión arterial (HTA) e hiperlipidemia. Además se recogió información sobre la extensión de la enfermedad coronaria (EEC, definida por la presencia de estenosis mayor del $70 \%$ en al menos dos arterias coronarias), el éxito de la AP (definido por la consecución de un flujo TIMI grado 3 en la arteria responsable del infarto con una estenosis residual inferior al 50\%) $)^{17}$, la localización del infarto (expresada en función de la arteria responsable del mismo), así como su tiempo de evolución hasta la revascularización y la presencia de insuficiencia cardiaca (IC) al ingreso (diagnosticada según la clasificación clínica de Killip y Kimball $)^{18}$. En este sentido la variable IC se expresó como una variable dicotómica en función de que la clasificación de Killip y Kimball al ingreso de cada paciente fuera $\geq 2$ para establecer su diagnóstico frente a los pacientes con grado 1 y por tanto sin IC. No se especificó la distribución de los pacientes en ambos grupos para los diferentes grados de IC según dicha clasificación por el limitado tamaño muestral y el relativamente escaso número de pacientes en cada subgrupo, lo que no permitió establecer comparaciones de significación estadística. También se analizó en ambos grupos el tratamiento farmacológico recibido durante la hospitalización (que incluyó tratamiento con ácido acetilsalicílico, clopidogrel, heparina de bajo peso molecular, bloqueadores beta, estatinas, inhibidores de la enzima convertidora de angiotensina [IECA] y diuréticos), los niveles máximos en plasma de troponina I y la fracción de eyección ventricular 
TABLA 1. Características clínicas basales del grupo de hombres y del grupo de mujeres

\begin{tabular}{|c|c|c|c|}
\hline & Hombres $(\mathrm{n}=52)$ & Mujeres $(\mathrm{n}=34)$ & Valor de $\mathrm{p}$ \\
\hline Edad (media \pm DE) en años & $65 \pm 11$ & $70 \pm 8$ & 0,02 \\
\hline Tabaquismo & $34 \%$ & $22 \%$ & 0,001 \\
\hline Diabetes mellitus & $18 \%$ & $37 \%$ & 0,002 \\
\hline HTA & $33 \%$ & $58 \%$ & $<0,001$ \\
\hline Dislipidemia & $35 \%$ & $29 \%$ & 0,34 \\
\hline Estenosis $>70 \%$ en 2 vasos & $18 \%$ & $23 \%$ & 0,23 \\
\hline Éxito del procedimiento & $90 \%$ & $94 \%$ & 0,07 \\
\hline Killip $\geq 2$ al ingreso & $12 \%$ & $22 \%$ & 0,01 \\
\hline Mortalidad intrahospitalaria & $8 \%$ & $17 \%$ & 0,002 \\
\hline
\end{tabular}

DE: desviación estándar; HTA: hipertensión arterial.

izquierda (FEVI) postrevascularización. Finalmente se recogió la mortalidad intrahospitalaria (en los primeros 28 días tras el ingreso) en los grupos de estudio.

Todas las variables anteriormente expuestas fueron recogidas por los dos cardiólogos habitualmente dedicados a la asistencia de la Unidad Coronaria de nuestro hospital. Todos los pacientes dieron su consentimiento informado por escrito previamente a la inclusión en el estudio y el protocolo de estudio fue aprobado por el comité ético de nuestro hospital.

\section{Análisis estadístico}

Para el análisis de los datos se utilizó el paquete estadístico SPSS (Statistical Package for Social Sciences, versión 10.0 para Windows). En relación a la estadística descriptiva, las variables cualitativas se expresaron como porcentajes, mientras que las cuantitativas se expresaron como media \pm la desviación estándar. La proximidad a la distribución normal de nuestra población fue evaluada mediante la prueba de Kolmogorov-Smirnov. Para la comparación entre las variables cualitativas se empleó la prueba de la $\chi^{2}$. La comparación entre variables cuantitativas se realizó mediante la prueba de la t de Student. Finalmente se realizó un análisis de regresión logística con el método introducir para determinar las variables predictoras independientes de mortalidad intrahospitalaria. Con el tamaño muestral de este trabajo, 52 hombres y 34 mujeres ( $\mathrm{N}=86$ pacientes), obtuvimos una potencia estadística para nuestro estudio del $33,9 \%$ para las conclusiones que posteriormente se presentarán. Un valor de $\mathrm{p}<0,05$ se consideró estadísticamente significativo.

\section{RESULTADOS}

Del total de la población de estudio (86 pacientes), 52 eran hombres y 34 mujeres. Las características basales de ambos grupos se muestran en la tabla 1. El grupo de mujeres presentó significativamente mayor edad y mayor prevalencia de diabetes mellitus (DM) y de hipertensión arterial (HTA). Sin embargo, el grupo de hombres presentó mayor incidencia de tabaquismo. En el resto de las características basales no se observaron diferencias significativas. Como se muestra en la tabla 2, no hubo diferencias significativas en la localización del infarto y su tiempo de evolución entre ambos grupos. Asimismo tampoco hubo diferencias en relación a los niveles de troponina I y la FEVI posrevascularización entre ambos grupos. En cuanto al tratamiento médico empleado durante la hospitalización, fue similar en ambos grupos, con excepción de los diuréticos e IECA, más comúnmente empleados en las mujeres ( $\mathrm{p}=0,03$ ).

El grupo de mujeres presentó de forma significativa una mayor incidencia de IC al ingreso y una mayor mortalidad intrahospitalaria en los primeros 28 días tras el ingreso que el de hombres, como se muestra en la tabla 1. Tras ajustar por los posibles factores de confusión, la edad y los factores de riesgo cardiovascular, en el análisis de regresión logística múltiple el sexo femenino (odds ratio [OR]: 2,1 ; intervalo de confianza [IC] del 95\%: 1,3-2,5; p < 0,001) y la IC al ingreso (OR:1,8; IC del 95\%: 1,1-2,0; $\mathrm{p}<0,01$ ) permanecieron como predictores independientes de mortalidad intrahospitalaria.

\section{DISCUSIÓN}

El principal hallazgo de este trabajo fue que el sexo femenino se asoció de forma independiente con una mayor mortalidad intrahospitalaria en los primeros 28 días tras un IAMEST revascularizado con AP. Nuestros resultados coinciden con los de otros trabajos previos $^{7,9-11,19-21}$. Además las mujeres de nuestra población presentaron significativamente una mayor incidencia de IC al ingreso que los hombres, como también han publicado otros autores $s^{6-8,15,20}$. Finalmente, en nuestra serie, ambas variables se mostraron como predictores de mortalidad intrahospitalaria ${ }^{7,9-11,19-21}$ con independencia de que todos los pacientes fueran revascularizados mediante AP.

En relación con las características basales, en el presente estudio las mujeres tenían significativamente más edad que los hombres. Además, el grupo de mujeres presentó mayor prevalencia de comorbilidades tales como HTA y DM. Sin embargo, el grupo de hombres tuvo mayores tasas de tabaquismo que el de mujeres. No hubo diferencias en relación a la prevalencia de dislipidemia ni a la EEC. Estos hallazgos concuerdan con los observados por la mayoría de los trabajos previos ${ }^{7,9-11,19-22}$. En este sentido, la mayor pre- 
FERRER-HITA JJ ET AL. EL GÉNERO FEMENINO ES UN PREDICTOR INDEPENDIENTE DE LA MORTALIDAD INTRAHOSPITALARIA EN PACIENTES CON INFARTO AGUDO DE MIOCARDIO CON ELEVACIÓN DEL SEGMENTO ST REVASCULARIZADO

MEDIANTE UNA ANGIOPLASTIA PRIMARIA

TABLA 2. Tratamiento farmacológico recibido durante la hospitalización, niveles pico de troponina I, fracción de eyección ventricular izquierda posrevascularización y tiempo hasta la revascularización y localización del infarto en ambos grupos

\begin{tabular}{|c|c|c|c|}
\hline & Hombres $(\mathrm{n}=52)$ & Mujeres $(n=34)$ & Valor de $\mathrm{p}$ \\
\hline Ácido acetilsalicílico & $100 \%$ & $100 \%$ & 1 \\
\hline Clopidogrel & $94 \%$ & $100 \%$ & 0,88 \\
\hline Bloqueadores beta & $75 \%$ & $68 \%$ & 0,76 \\
\hline НВРМ & $100 \%$ & $100 \%$ & 1 \\
\hline Estatinas & $100 \%$ & $100 \%$ & 1 \\
\hline IECA & $62 \%$ & $80 \%$ & 0,03 \\
\hline Diuréticos & $20 \%$ & $39 \%$ & 0,03 \\
\hline Troponina I (pico sérico) & $70 \pm 20$ & $65 \pm 17$ & 0,23 \\
\hline FEVI posrevascularización & $51 \pm 7 \%$ & $48 \pm 10 \%$ & 0,10 \\
\hline \multicolumn{4}{|l|}{ Tiempos de retraso (minutos) } \\
\hline Síntomas-admisión & $240 \pm 55$ & $226 \pm 43$ & 0,21 \\
\hline Admisión-inflado balón & $45 \pm 15$ & $40 \pm 12$ & 0,10 \\
\hline \multicolumn{4}{|l|}{ Arteria reponsable del IAM: } \\
\hline Arteria descendente anterior & $55 \%$ & $40 \%$ & 0,06 \\
\hline Arteria circunfleja & $10 \%$ & $15 \%$ & 0,24 \\
\hline Arteria coronaria derecha & $35 \%$ & $45 \%$ & 0,09 \\
\hline
\end{tabular}

FEVI: fracción de eyección ventricular izquierda; HBPM: heparina de bajo peso molecular; IAM: infarto agudo de miocardio; IECA: inhibidores de la enzima convertidora de angiotensina.

sencia de comorbilidades en las mujeres se ha propuesto como justificación de su peor pronóstico a corto plazo tras un IAMEST, tal y como también hallamos en nuestro trabajo. Sin embargo, la elevada mortalidad intrahospitalaria en el grupo de mujeres se mantuvo tras el ajuste por estas variables, lo que sugiere que dichas comorbilidades asociadas sólo contribuirían parcialmente a este peor pronóstico tras un IAMEST y apoyaría la condición del sexo femenino como predictor independiente de mortalidad intrahospitalaria ${ }^{7,10,11,15}$.

Por otro lado, se siguen publicando trabajos que recogen que el abordaje terapéutico de la cardiopatía isquémica en las mujeres difiere negativamente del de los hombres, con tasas menores de empleo de tratamientos antiagregantes, terapia con bloqueadores beta y estrategias de reperfusión ${ }^{6-8,10,15,19-22}$, lo que podría influir en su peor pronóstico. En este sentido, el hecho de que en nuestro trabajo todos lo pacientes fueran revascularizados con $\mathrm{AP}$, sin diferencias en el éxito de la reapertura del vaso coronario entre sexos, junto con la homogeneidad del tratamiento farmacológico entre ambos grupos, apoya el aspecto diferencial independiente que obtuvimos sobre la mortalidad intrahospitalaria tras un IAMEST en función del sexo. La mayoría de las series publicadas, aunque indudablemente mayores que nuestra serie, no emplearon sistemáticamente en todos los pacientes la AP como estrategia de reperfusión coronaria (a día de hoy claramente superior a la reperfusión farmacológica), cosa que sí se garantizó en nuestro trabajo ${ }^{6-8,10,19-22}$. Creemos que quizás en ello puede fundamentarse la importancia de nuestros hallazgos, pese a que la muestra poblacional evidentemente no tiene el tamaño deseable.

Junto a todo lo anterior, se han propuesto además otras explicaciones para justificar este peor pronóstico en las mujeres tras un IAMEST. Así, es conocido que la clase Killip y Kimball tras un IAMEST es uno de los predictores más potentes de mortalidad ${ }^{18}$ y las mujeres de nuestra población presentaron significativamente mayor grado de IC al ingreso que los hombres. De acuerdo con lo publicado no hubo diferencias en la fracción de eyección tras el IAMEST entre grupos, lo que sugiere que dicha IC vino mediada fundamentalmente por una mayor presencia de disfunción diastólica en el grupo de mujeres ${ }^{6,10,15}$. Probablemente esto venga explicado por la existencia de una menor reserva compensatoria diastólica en las mujeres, como se ha hipotetizado ${ }^{15}$. Además, se ha descrito una mayor incidencia de complicaciones mecánicas tras un IAMEST en las mujeres que en los hombres ${ }^{15}$, aunque en nuestra serie no se recogió específicamente la aparición de las mismas, sino globalmente la mortalidad intrahospitalaria (en los 28 días posteriores al ingreso), sin analizar la etiología concreta de cada fallecimiento. Probablemente esto infraestimaría la mortalidad pasados estos 28 días, si bien el análisis de esta no fue motivo de este estudio.

Desde el punto de vista fisiopatológico se han considerado también aspectos diferenciales asociados al sexo para justificar este peor pronóstico. Así, se ha sugerido que las mujeres pueden tener arterias coronarias de menor calibre y menor circulación colateral que los hombres en relación a su menor superficie corporal. Además, los estados de hipercoagulabilidad y el espasmo coronario, mecanismos descritos en mujeres jóvenes, así como una mayor susceptibilidad genética, también pueden desempeñar un papel en este peor pronóstico asociado al sexo femenino ${ }^{7,15}$.

Como ya comentamos, la principal limitación de nuestro trabajo viene condicionada por el relativamente escaso número de pacientes que conforman el tamaño muestral. Ya hemos comentado que con dicho tamaño nuestro trabajo presenta una potencia estadística del 33,9\% para las conclusiones que obtenemos, lo cual es claramente mejorable. Sería necesario diseñar estudios multicéntricos con mayor número de 
pacientes y con estrategias terapéuticas homogéneas para ambos sexos para poder confirmar nuestros hallazgos.

En definitiva, y a tenor de nuestros resultados, podemos concluir que en nuestra serie, el sexo femenino se asoció de forma independiente con una mayor mortalidad intrahospitalaria tras un IAMEST revascularizado con AP. Estos hallazgos, junto al emergente interés que vuelve a cobrar el tema de la cardiopatía isquémica en la mujer en muchos aspectos ${ }^{6,22}$, hacen que creamos necesario volver a profundizar en este tema con nuevos estudios con mayor número de pacientes para establecer definitivamente cuáles son los aspectos diferenciales del sexo en relación al pronóstico tras un IAMEST. De esta forma, todos lo profesionales implicados en la atención de la cardiopatía isquémica aguda y crónica estaremos más concienciados y seremos más eficaces en el servicio que prestamos.

\section{Declaración de conflicto de intereses}

Los autores han declarado no tener ningún conflicto de intereses.

\section{BIBLIOGRAFÍA}

1. Mosca L, Appel LJ, Benjamin EJ, Berra K, Chandra-Strobos N, Fabunmi RP, et al. Evidence-based guidelines for cardiovascular disease prevention in women. Circulation. 2004;109:672-93.

2. Mosca L, Ferris A, Fabunmi R, Robertson RM. Tracking women's awareness of heart disease. An American Heart Association Nacional Study. Circulation. 2004;109:573-9.

3. Heindenreich P, McClellan M. Trends in treatment and outcomes for acute myocardial infarction: 1975-1995. Am J Med. 2001;110:165-74

4. Rosamond WD, Chambless LE, Folsom AR, Cooper LS Conwill DE, Clegg L, et al. Trends in the incidence of myocardial infarction and in mortality due to coronary heart disease, 1987 to 1994. N Engl J Med. 1998;339:861-7.

5. Heras M, Marrugat J, Arós F, Bosch X, Enero J, Suárez MA, et al. Reducción de la mortalidad por infarto agudo de miocardio en un periodo de 5 años. Rev Esp Cardiol. 2006;59:200-8.

6. Alfonso F, Bermejo J, Segovia J. Enfermedades cardiovasculares en la mujer: ¿por qué ahora? Rev Esp Cardiol. 2006;59:259-63.

7. Vaccarino V, Parsons L, Every NR, Barron HV, Krumholz HM. Sex-based differences in early mortality after myocardial infarction. N Engl J Med 1999;341:217-25.

8. Gan SC, Beaver SK, Houck PM, MacLehose RF, Lawson HW, Chan L. Treatment of acute myocardial infarction and 30-day mortality among women and men. N Engl J Med. 2000;343:8-15.
9. Greenland P, Reicher-Reiss H, Goldbourt U, Behar S. In-hospital and 1-year mortality in 1524 women after myocardial infarction: comparison with 4315 men. Circulation. 1991;83:484-91.

10. Marrugat J, Sala J, Masiá R, Pavesi M, Sanz G, Valle V, et al. Mortality differences between men and women following first myocardial infarction. RESCATE Investigators. Recursos Empleados en el Síndrome Coronario Agudo y Tiempo de Espera. JAMA. 1998;280:1405-9.

11. Ceniceros Rozalén I, Gastaldo Simeón R, Cabadés O'Callaghan A, Cebrián Doménech J. El sexo femenino es un factor pronóstico independiente de mortalidad en la fase aguda del infarto de miocardio. Med Clin (Barc). 1997;109:171-4.

12. Dittrich H, Gilpin E, Nicod P, Cali G, Henning H, Ross J Jr. Acute myocardial infarction in women: influence of gender on mortality and prognostic variables. Am J Cardiol. 1988;62:1-7.

13. Stone GW, Grines CL, Browne KF, Marco J, Rothbaum D, O'Keefe J, et al. Predictors of in-hospital and 6-month outcome after acute myocardial infarction in the reperfusion era: the Primary Angioplasty in Myocardial Infarction (PAMI) trial. J Am Coll Cardiol. 1995;25:370-7.

14. Stone GW, Grines CL, Browne KF, Marco J, Rothbaum D, O'Keefe J, et al. Comparison of in-hospital outcome in men versus women treated by either thrombolytic therapy or primary coronary angioplasty for acute myocardial infarction. Am J Cardiol. 1995; 75:987-92.

15. Marrugat J, Sala J, Aboal J. Epidemiología de las enfermedades cardiovasculares en la mujer. Rev Esp Cardiol. 2006;59: 264-74.

16. Van de Werf F, Ardissino D, Betriu A, Cokkinos DV, Falk E, Fox KA, et al. Management of acute myocardial infarction in patients presenting with ST-segment elevation. The Task Force on the Management of Acute Myocardial Infarction of the European Society of Cardiology. Eur Heart J. 2003;24:28-66.

17. The TIMI Study Group. The Thrombolysis In Myocardial Infarction (TIMI) trial. Phase I findings. TIMI Study Group. N Engl J Med. 1985;312:932-6.

18. Killip T, Kimball JT. Treatment of myocardial infarction in a coronary care unit: a two-year experience with 250 patients. Am J Cardiol. 1967;20:467-74.

19. Karcioglu Ö, Ünal Aslan B, Aslan O. Gender differences in the management and survival of patients with acute myocardial infarction. Eur J Intern Med. 2002;13:474-9.

20. Marrugat J, García M, Elosua R, Aldasoro E, Tormo MJ, Zurriaga O, et al. Short-term ( 28 days) prognosis between genders according to the type of coronary event (Q-wave versus non-Q-wave acute myocardial infarction versus unstable angina pectoris). Am J Cardiol. 2004;94:1161-5.

21. Maynard C, Litwin PE, Martin JS, Weaver WD. Gender differences in the treatment and outcome of acute myocardial infarction: results from the Myocardial Infarction Triage and Intervention Registry. Arch Intern Med. 1992;152:972-6.

22. Conthe Gutiérrez P, Lobos Bejarano JM, González Juanatey JR, Gil Núñez A, Pajuelo Fernández FJ, Novials Sardá A, et al. Diferencias en la atención de las mujeres con alto riesgo cardiovascular respecto a los varones: estudio multidisciplinario. Med Clin (Barc). 2003;120:451-5. 